\title{
Effects of molecular structure on the physical, chemical, and electrical properties of ester-based transformer insulating liquids
}

\begin{abstract}
This article presents the experimental studies carried out on the environmental friendly polyol ester insulating liquids to investigate the effect of molecular structure on the physical, chemical, and electrical properties. Six different polyol esters that can be produced from the transesterification of various methyl esters with neopentylglycol/trimethylolpropane alcohols were synthesized and compared with those of refined, bleached, and deodorized palm oil (RBDPO) and mineral transformer oil. The finding suggests that the physical properties like fire point, pour point, and viscosity are very much affected by the molecular weight and molecular composition of the polyol esters. The electrical properties are also highly influenced by the molecular structure-related characteristics, such as the polarity, dipole polarization, carbon chain length, and degree of branching. The results confirm the findings of previous studies that the polyol esters and RBDPO have more polarity and dipole polarization compared to mineral oil. The experimental evidence showed that the newly synthesized insulating liquids have favorable thermal and electrical properties, thus suggesting that the insulating liquids have the potential to replace conventional insulating liquids to provide a more sustainable power system in the future.
\end{abstract}

Keyword: Transformers; Insulating liquids; Natural esters; Polyol esters; Molecular structure; Electrical properties; Transesterification 